\title{
Improvement of Messages Delivery Time on Vehicular Delay-Tolerant Networks
}

\author{
Vasco N. G. J. Soares \\ Instituto de \\ Telecomunicações \\ Univ. of Beira Interior \\ Pol. Inst. Castelo Branco \\ Castelo Branco, Portugal \\ vasco.g.soares@ieee.org
}

\author{
Joel J. P. C. Rodrigues \\ Instituto de \\ Telecomunicações \\ Department of Informatics \\ Univ. of Beira Interior \\ Covilhã, Portugal \\ joeljr@ieee.org
}

\author{
Paulo Salvador Ferreira \\ Instituto de \\ Telecomunicações \\ University of Aveiro \\ Aveiro, Portugal \\ salvador@ua.pt
}

\author{
António M. D. Nogueira \\ Instituto de \\ Telecomunicações \\ University of Aveiro \\ Aveiro, Portugal \\ nogueira@ua.pt
}

\begin{abstract}
Vehicular Delay-Tolerant Networks (VDTNs) are an application of the Delay-Tolerant Network (DTN) concept, where the movement of vehicles and their message relaying service is used to enable network connectivity under unreliable conditions. To address the problem of intermittent connectivity, long-term message storage is combined with routing schemes that replicate messages at transfer opportunities. However, these strategies can be inefficient in terms of network resource usage. Therefore, efficient scheduling and dropping policies are necessary to improve the overall network performance. This work presents a performance analysis, based on simulation, of the impact of different scheduling and dropping policies enforced on Epidemic and Spray and Wait routing schemes. This paper evaluates these policies from the perspective of their efficiency in reducing the message's end-to-end delay. In our scenario, it is shown that when these policies are based on the message's lifetime criteria, the message average delay decreases significantly and the overall message delivery probability also increases for both routing protocols. Further simulations show that these results outperform the MaxProp and PRoPHET routing protocols that have their own scheduling and dropping mechanisms.
\end{abstract}

Keywords-Delay/Disruption-Tolerant Networks; Vehicular Networks; Scheduling Algorithms; Dropping Policies

\section{INTRODUCTION}

The Delay-Tolerant Networking (DTN) architecture [1] is built as an overlay network, based on a store-and-forward strategy, to provide communication facilities in challenged environments where sparse intermittent connectivity, high latency, asymmetric data rates, high loss rates, and even no end-to-end connectivity exist. In DTNs, messages (bundles) of arbitrary size may be stored for long periods of time at network nodes, and are conveyed hop-by-hop to the destination.

DTNs have been widely applied to various areas including interplanetary networks [2], underwater networks [3], wildlife tracking sensor networks [4], and networks to benefit developing communities [5]. Vehicular DelayTolerant Networking (VDTN) is a particular application of a mobile DTN concept characterized by opportunistic contacts, where vehicle mobility is used for connectivity.
VDTNs have several application scenarios including: traffic condition monitoring, collision avoidance, emergency message dissemination, free parking spots information, advertisements and, for example, to gather data collected by vehicles (like road pavement defects) [6-9].

Figure 1 shows an example of a VDTN. Mobile nodes (e.g., vehicles) physically carry data, exchanging information with one another. They move along the roads randomly (e.g. cars), or following predefined routes (e.g. buses). Stationary relay nodes are fixed devices located at crossroads, with store-and-forward capabilities. They allow mobile nodes passing by to pickup and deposit data on them, thus increasing the number of contact opportunities.

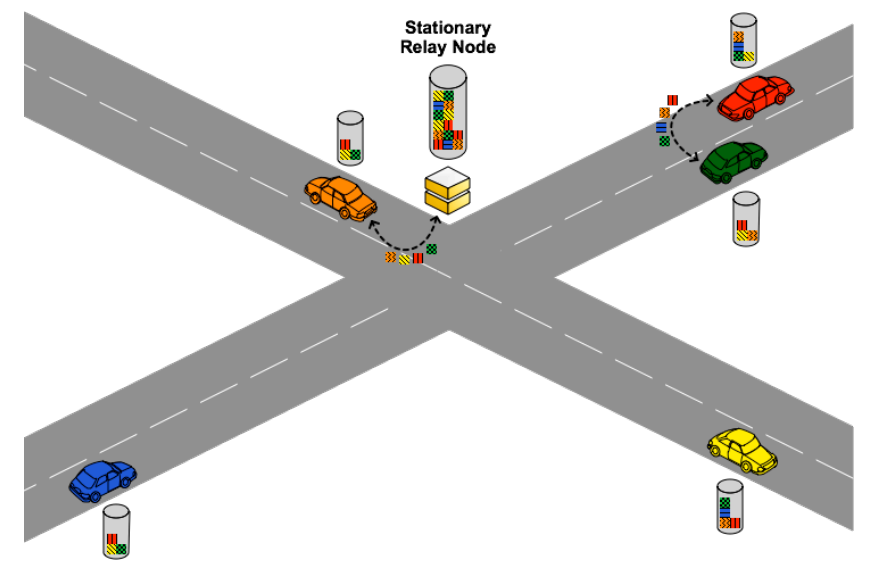

Figure 1. Example of Vehicular Delay-Tolerant Network nodes.

To cope with intermittent connectivity, VDTN network nodes store messages on their buffers, while waiting for opportunities to forward messages to intermediate nodes or to the final destination. Routing protocols replicate messages along multiple network nodes (paths) to improve their delivery probability and minimize the message delivery delay. In a resource-constrained network, the combination of message storage during large periods of time and their replication degrades the overall network performance and motivates the need for effective scheduling and dropping policies. 
Depending on the VDTN application scenario, it can be more important to maximize the delivery ratio (e.g. an application for environmental pollution data collection), or to minimize the delivery delay (e.g. an application for advertisements or traffic notification). In the context of this work we are interested in evaluating the impact of scheduling and dropping policies on the reduction of the delivery delay. These policies will be enforced on two DTN routing protocols, the Epidemic [10] and the Spray and Wait [11]. We also compare their performance with MaxProp [12] and PRoPHET [13], two routing protocols that have their own scheduling and dropping mechanisms.

The remainder of the paper is organized as follows. Section II describes the problem and our approach. Section III presents the performance evaluation of our approaches in comparison with above-mentioned MaxProp and PRoPHET protocols. Finally, section IV concludes the paper.

\section{PROBLEM DESCRIPTION AND BACKGROUND}

Vehicle Delay-Tolerant Networks are characterized by high node mobility, which in conjunction with energy constraints, finite bandwidth, short radio transmission ranges or obstructed radio links, result in short contact duration times and intermittent connectivity. In order to increase message delivery ratio and reduce message average delay, multiple message replicas can be propagated and stored in the network. However, these flooding-based routing approaches degrade their performance when network resources (e.g. bandwidth, and storage) are limited. Therefore, efficient scheduling and dropping policies are necessary to improve the end-to-end performance of the network.

The scheduling policy determines the order by which messages should be sent during data exchange between network nodes. The dropping policy determines which messages are dropped when buffer overflow occurs. Figure 2 illustrates the scheduling and dropping policies considered in this study.

FIFO scheduling policy orders messages to be forwarded at a contact opportunity based on their receiving time (first-come, first-served basis). Hence, there is no guarantee that the TTL of these messages will expire soon or not. Random scheduling policy sends messages in a random order. Lifetime DESC (Descending Order) scheduling policy orders messages based on time-to-live (TTL). Messages with longer TTLs will be scheduled to be sent first. Since messages exchanged between network nodes will have longer remaining lifetimes, this increases their probability to be relayed more times between network nodes, until eventually reaching the destination (and before expiring).

When a FIFO dropping policy is enforced, in cases of buffer congestion dropped messages will be the ones at the head of the queue ("drop head"). So, there is no guarantee that the remaining TTL of these messages is smaller than the TTL of any other message that is stored on the buffer. A Lifetime ASC (Ascending Order) dropping policy guarantees that the messages selected to be dropped will be the ones whose remaining TTL expires sooner, and therefore have less time left to reach destination before expiring.

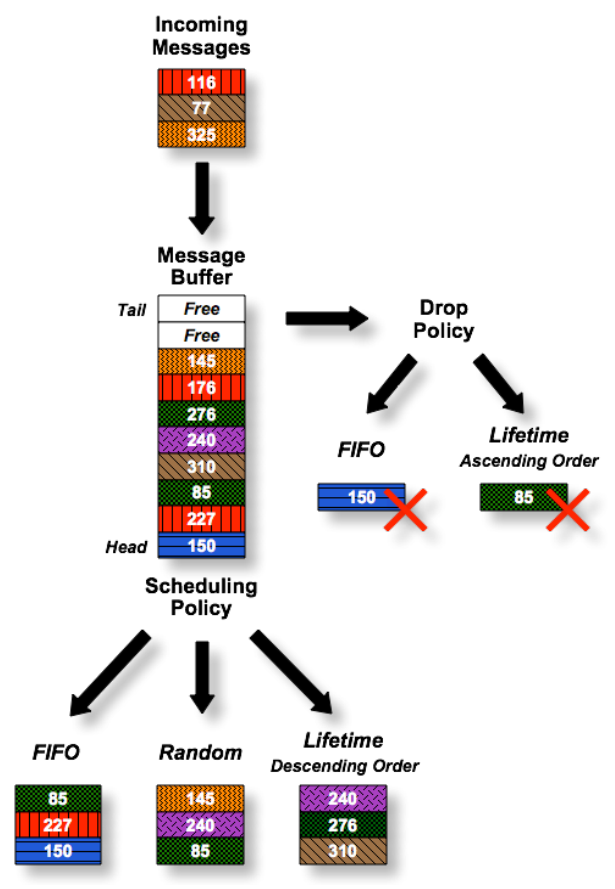

Figure 2. Scheduling and dropping policies considered in this study. The value inside the messages represents their remaining time-to-live.

In this work, we evaluate the impact that enforcing the above-mentioned policies on two well-known DTN routing protocols, Epidemic and Spray and Wait, will have on the performance of VDTN networks. Epidemic is a floodingbased routing protocol where nodes exchange the messages they don't have. In an environment with infinite buffer space and bandwidth, this protocol performs better than the other ones in terms of message delivery ratio and latency, providing an optimal solution. However, its naive flooding wastes resources and severely degrades the overall performance when resources are limited.

Spray and Wait creates a number of copies $N$ to be transmitted ("sprayed") per message (assuming 12, in this study). In its binary variant (considered in this work), any node $A$ that has more than 1 message copies and encounters any other node $B$ that doesn't have a copy, forwards to $B$ $N / 2$ message copies and keeps the rest of the messages. A node with 1 copy left, only forwards it to the final destination.

We turn our attention to the combination of a Lifetime based scheduling and dropping policy. Then, we intend to evaluate if they will reduce the average delivery delay considerably, and what effects this policy will have over the 
message delivery ratio. Additionally, we compare these results to the ones obtained with the MaxProp and PRoPHET routing protocols.

MaxProp prioritizes the schedule of messages transmitted to other nodes and also the schedule of messages to be dropped. Historical data of path probabilities to nodes, acknowledgments, head-start mechanism, and lists of previous intermediaries, are used to calculate the priorities.

PRoPHET is a probabilistic routing protocol that considers a history of encounters and transitivity. It considers that nodes move in a non-random pattern, and applies "probabilistic routing". PRoPHET also has its own schedule and discard policies. In this work, we consider PRoPHET with the GRTRMax forwarding strategy (policy).

\section{PERformance EVAluation}

This section studies the impact of the above described scheduling and dropping policies on the performance of a VDTN network. The simulation tool used in this study is the Opportunistic Network Environment (ONE) Simulator [14]. We developed a set of extensions to the simulator to implement the Lifetime based scheduling and dropping policies.

For the simulation scenario, we use a map-based model of a small part of the city of Helsinki (Figure 3). We simulate 40 vehicles, each with a 100 Mbytes message buffer, moving across the map roads. Once a vehicle reaches a destination, it randomly waits 5 to 15 minutes. Then, it selects a new random map location, and a random speed between 30 and $50 \mathrm{~km} / \mathrm{h}$. The vehicle moves to the new destination using the shortest available path.

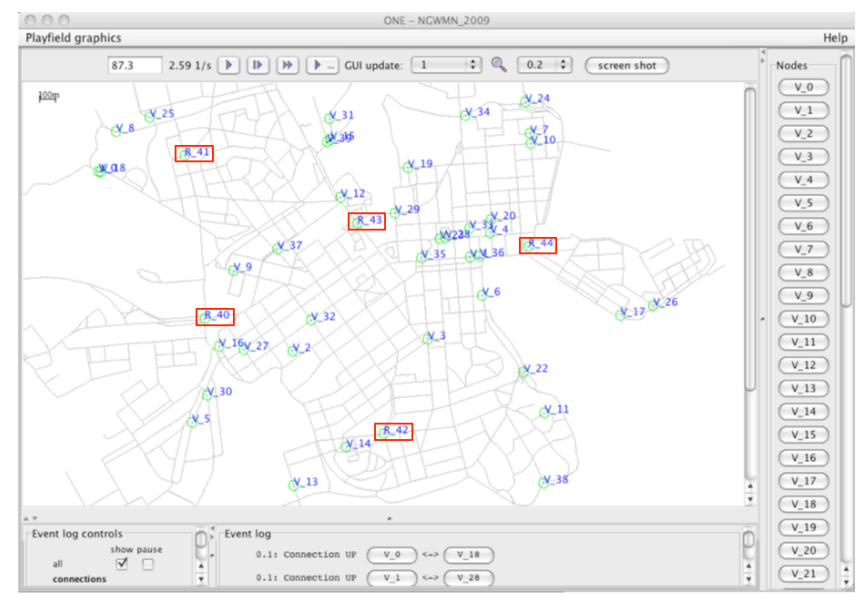

Figure 3. ONE Simulator running Helsinki simulation scenario (vehicles $\mathrm{V}$, relay nodes $\mathrm{R}$ ).

Five stationary relay nodes are placed at the predefined map locations shown in Figure 3 (rectangles presented in the figure). Each one has a 500 Mbytes message buffer size. Network nodes connect to each other using IEEE 802.11b with a data rate of $6 \mathrm{Mbit} / \mathrm{s}$ and a transmission range of 30 meters, using omni-directional antennas. Vehicles exchange messages with each other and with relay nodes.

Messages have random source and destination vehicles, and are generated using an inter-message creation interval that is uniformly distributed in the range of $[15,30]$ (seconds). Message size is uniformly distributed in the range of [500 K, $2 \mathrm{M}$ ] (Bytes). Messages are deleted from buffers when congestion occurs, or when their TTL expires. When a node delivers a message to its final destination, that message is discarded from the sender node's buffer.

The message time-to-live (TTL) changes between 60, 90, 120,150 , and 180 minutes, along the simulations. Increasing the TTL value of the messages will make us have more messages stored at the network nodes' buffers and during larger periods of time. Therefore, more messages will be exchanged between network nodes, and this will also potentially cause buffer overflows. Hence, scheduling and dropping policies will have a major role to improve the overall performance of VDTN in terms of message average delay and message delivery probability.

We measure the performance differences when the combination of the scheduling and dropping policies, presented at Table I, is enforced. We assume a cooperative opportunistic environment without knowledge of the traffic matrix and contact opportunities, simulating a 12-hour period.

TABLE I. COMBINED SCHEDULING - DropPING Policies

\begin{tabular}{|c|}
\hline Scheduling - Dropping \\
\hline FIFO - FIFO \\
\hline Random - FIFO \\
\hline $\begin{array}{c}\text { Lifetime DESC - Lifetime ASC } \\
\text { (Descending order) (Ascending order) }\end{array}$ \\
\hline
\end{tabular}

Performance metrics considered in this study are the message delivery delay (measured as the time between message creation and delivery), and the message delivery probability (measured as the relation of the number of unique delivered messages to the number of messages sent).

\section{A. Performance Assessment of Epidemic Routing Protocol}

We start our evaluation focusing on results observed when Epidemic routing protocol is used. The FIFO-FIFO policy with its scheduling and dropping message selection criteria based in the order of message arrival to the buffer, presents the worst performance in terms of message average delay and message delivery probability across all simulations, as it can be observed in Figures 4 and 5.

The Random-FIFO policy introduces a slight modification on the previous policy, because messages are scheduled for transmission in a random order. As a result of this simple adjustment, the message average delay 
decreased, and the delivery ratio increased. When comparing with the FIFO-FIFO policy, Figure 4 shows that messages arrive at the destination nodes approximately 2, 4, 6,8 , and 8 minutes sooner in average, and Figure 5 shows that delivery probability increased in $2 \%, 4 \%, 4 \%, 3 \%$, and $3 \%$, respectively.

It is interesting to notice that deploying a Lifetime DESC-Lifetime ASC based policy and, therefore, scheduling and dropping messages based on their remaining lifetime, not only results in a decrease of the message average delay, as expected, but also contributes to improve the delivery ratio (Figures 4 and 5). As expected, Figure 4 shows that this policy presents the best results across all simulations, contributing to significantly decrease the message average delay. When compared to the FIFO-FIFO policy, messages arrive at the destination nodes approximately $6,12,19,25$, and 29 minutes sooner, in average. It can be observed in Figure 5 that this policy also increases the probability that messages reach the destination nodes, performing better than other studied policies. It presents gains of $9 \%, 11 \%$, $9 \%, 7 \%$ and $5 \%$, respectively, when compared to the $F I F O$ FIFO policy.

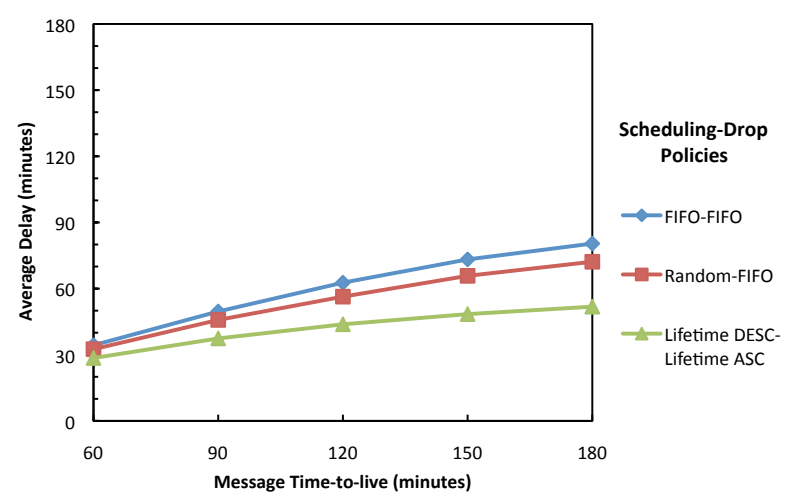

Figure 4. Message average delay using the Epidemic routing protocol.

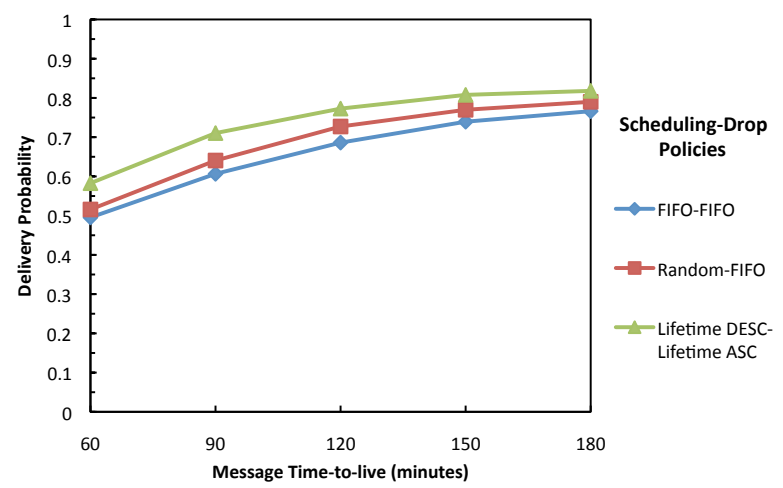

Figure 5. Message delivery probability using the Epidemic routing protocol.

\section{B. Performance Assessment of Spray and Wait Routing Protocol}

In this subsection we analyze the Spray and Wait protocol (binary variant). Contrary to Epidemic, Spray and Wait limits the number of copies of a message. This will cause less bandwidth utilization, and less congestion at the network nodes' buffers. Nevertheless, like on Epidemic, enforcing a Lifetime DESC-Lifetime ASC schedulingdropping policy on Spray and Wait routing protocol will improve the overall VDTN performance in terms of message average delay and delivery ratio. Figure 6 shows that messages will arrive at the destination nodes approximately 4, 9, 14, 18, and 21 minutes sooner, in average, when compared to the $F I F O-F I F O$ policy.

Figure 7 confirms that selecting messages to be transmitted and dropped based on their remaining TTL will increase about $8 \%, 6 \%, 5 \%, 3 \%$ and $3 \%$ the message delivery probability, when compared to the FIFO-FIFO policy. As expected, the gains in this performance metric are attenuated when messages have a large TTL. This is due to the fact that network nodes have large buffers and can carry and exchange these messages during longer periods of time before expiring. However, increasing the TTL reinforces the improvement on average delay that was introduced by the Lifetime DESC-Lifetime ASC policy.

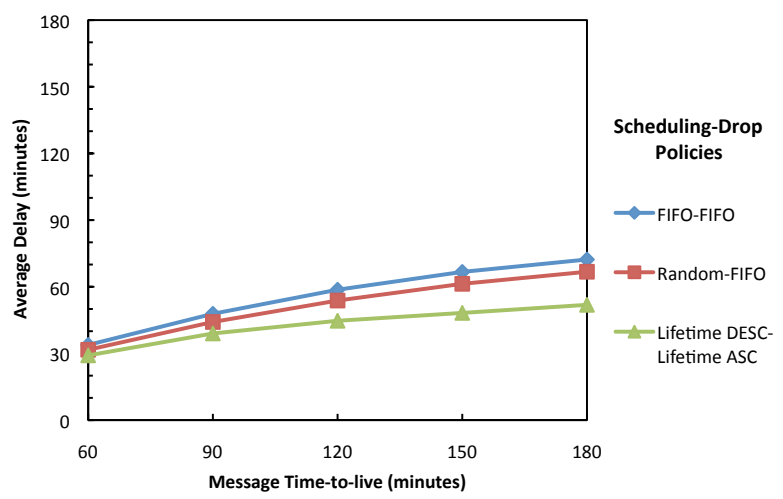

Figure 6. Message average delay using the Spray and Wait routing protocol.

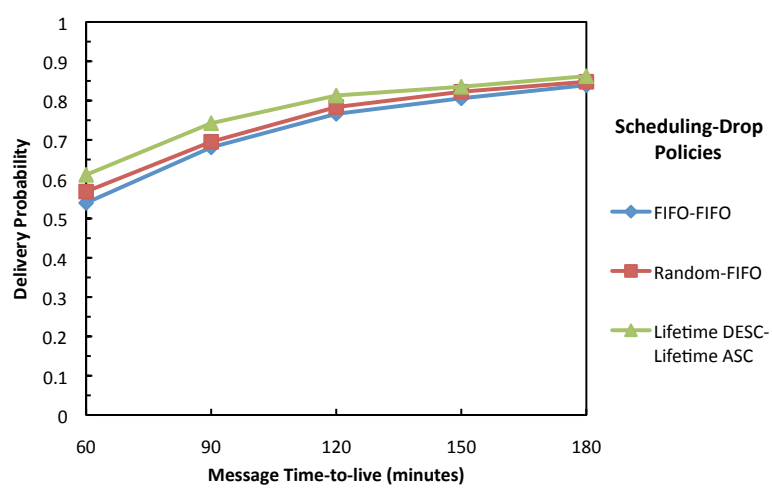

Figure 7. Message delivery probability using the Spray and Wait routing protocol. 


\section{Performance Assessment of MaxProp and PRoPHET Routing Protocols}

In this subsection, we evaluate the network performance of the MaxProp or PRoPHET routing protocols. We compare the performance of these protocols with the simpler Epidemic and Spray and Wait replicating strategies enforcing the above scheduling-dropping policy (Lifetime DESC-Lifetime ASC). Both MaxProp and PRoPHET use historic meeting information, and their own scheduling and discarding policies. Figures 8 and 9 illustrate the comparison between the message delivery probability and the message delivery delay for those four routing protocols.

An analysis of these figures shows that MaxProp only outperforms Spray and Wait message delivery probability when messages have a TTL equal or higher than 150 minutes (Figure 8 ). Nevertheless, only a very slight increase in the delivery ratio was registered for these cases. More importantly, it can also be concluded that MaxProp requires more time to deliver messages (Figure 9), even in the cases where its delivery ratio is lower than Spray and Wait.

PRoPHET registers the lowest delivery probabilities and the longest average delays across all simulations. Therefore, in our scenario, MaxProp and PRoPHET are outperformed by Spray and Wait.

Epidemic high buffer occupancy and high bandwidth utilization problems were largely attenuated by the small size of the messages, the large nodes' buffers and the low traffic demands that were considered in this study. A data transfer rate lower than the one considered would further limit the number of messages that could be successfully transmitted during a contact period. We believe that more constrained network resources would reinforce the performance impact of the above-evaluated policies.

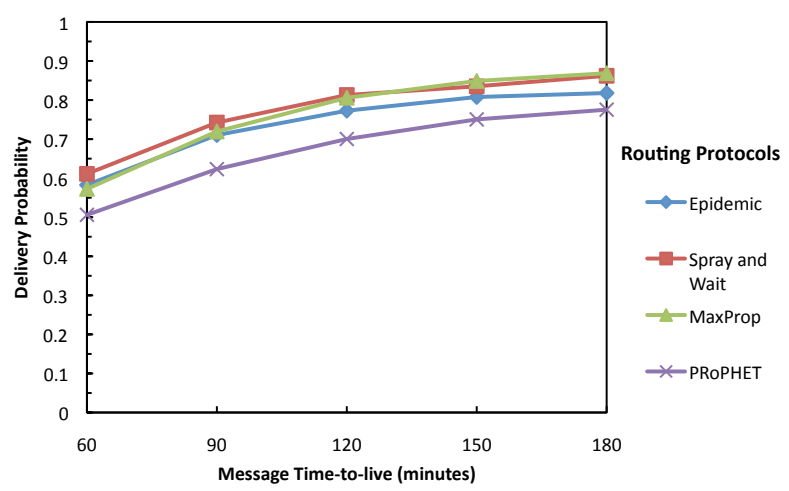

Figure 8. Comparison of the message delivery probability using the Epidemic, Spray and Wait, MaxProp, and PRoPHET routing protocols.

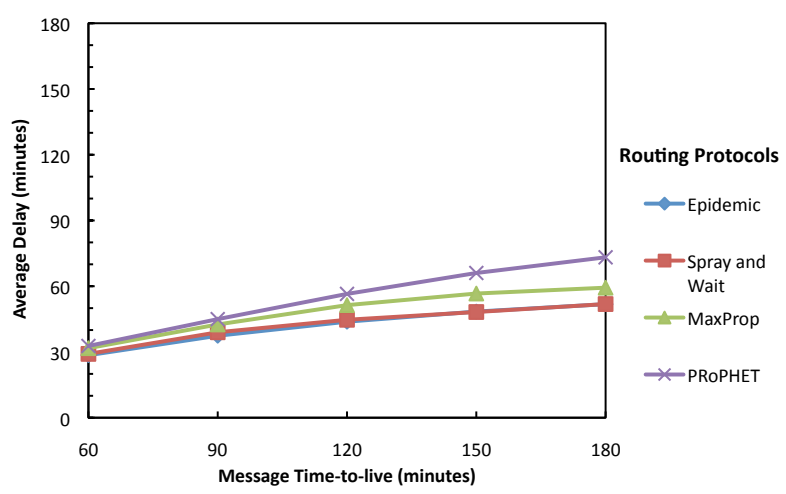

Figure 9. Comparison of the message average delay using the Epidemic, Spray and Wait, MaxProp, and PRoPHET routing protocols.

\section{CONCLUSIONS}

This paper evaluated the influence of scheduling and dropping policies on the performance of Vehicular DelayTolerant Networks. In this context, we looked for a combination of a scheduling and dropping policy that would minimize the delivery delay over the messages in the network. We also evaluated these policies from the perspective of their influence on the message delivery probability.

Simulation results with a real world map based model were presented and discussed. Different combinations of scheduling and dropping policies were enforced on the Epidemic and Spray and Wait DTN routing schemes. These protocols do not have native support for deciding the order by which messages should be sent or which messages should be discarded when there is buffer congestion. Results show a good performance obtained by the combination of scheduling and dropping policies based on the message's lifetime. In our simulation scenario, it was also observed that Spray and Wait could outperform MaxProp and PRoPHET scheduling and dropping mechanisms.

\section{ACKNOWLEDGMENTS}

Part of this work has been supported by Instituto de Telecomunicações, Next Generation Networks and Applications Group, Portugal, in the framework of the VDTN@Lab Project, and by the Euro-NF Network of Excellence from the Seventh Framework Programme of EU.

\section{REFERENCES}

[1] V. Cerf, S. Burleigh, A. Hooke, L. Torgerson, R. Durst, K. Scott, K. Fall, and H. Weiss, "Delay-Tolerant Networking Architecture," RFC 4838, April 2007, [Online]. Available: ftp://ftp.rfc-editor.org/innotes/rfc4838.txt.

[2] S. Burleigh, A. Hooke, L. Torgerson, K. Fall, V. Cerf, B. Durst, K. Scott, and H. Weiss, "Delay-Tolerant Networking: An Approach to Interplanetary Internet," in IEEE Communications Magazine, vol. 41, 2003, pp. 128-136.

[3] J. Partan, J. Kurose, and B. N. Levine, "A Survey of Practical Issues in Underwater Networks," in 1st ACM International Workshop on 
Underwater Networks, in conjunction with ACM MobiCom 2006, Los Angeles, California, USA, Sep. 25, 2006, pp. 17 - 24.

[4] P. Juang, H. Oki, Y. Wang, M. Martonosi, L. S. Peh, and D. Rubenstein, "Energy-Efficient Computing for Wildlife Tracking: Design Tradeoffs and Early Experiences with ZebraNet," ACM SIGOPS Operating Systems Review, vol. 36, pp. 96 - 107, 2002.

[5] A. Pentland, R. Fletcher, and A. Hasson, "DakNet: Rethinking Connectivity in Developing Nations," in IEEE Computer, vol. 37, 2004, pp. 78-83.

[6] R. Tatchikou, S. Biswas, and F. Dion, "Cooperative Vehicle Collision Avoidance using Inter-vehicle Packet Forwarding," in IEEE Global Telecommunications Conference (IEEE GLOBECOM 2005), St. Louis, MO, USA, 28 Nov. - 2 Dec., 2005.

[7] I. Leontiadis and C. Mascolo, "GeOpps: Geographical Opportunistic Routing for Vehicular Networks," in IEEE International Symposium on a World of Wireless, Mobile and Multimedia Networks 2007 (WoWMoM 2007), Espoo, Finland, 18-21 June, 2007, pp. 1-6.

[8] O. Brickley, C. Shen, M. Klepal, A. Tabatabaei, and D. Pesch, "A Data Dissemination Strategy for Cooperative Vehicular Systems," in Vehicular Technology Conference 2007 (VTC2007), Dublin, 2007, pp. 2501-2505.

[9] L. Franck and F. Gil-Castineira, "Using Delay Tolerant Networks for Car2Car Communications," in IEEE International Symposium on
Industrial Electronics 2007 (ISIE 2007), Vigo, Spain, 4-7 June, 2007, pp. 2573-2578.

[10] A. Vahdat and D. Becker, "Epidemic Routing for Partially-Connected Ad Hoc Networks," Duke University, Technical Report CS-2000-06, April, 2000.

[11] T. Spyropoulos, K. Psounis, and C. S. Raghavendra, "Spray and Wait: An Efficient Routing Scheme for Intermittently Connected Mobile Networks," in ACM SIGCOMM 2005 - Workshop on Delay Tolerant Networking and Related Networks (WDTN-05), Philadelphia, PA, USA, August 22-26, 2005.

[12] J. Burgess, B. Gallagher, D. Jensen, and B. Levine, "MaxProp: Routing for Vehicle-Based Disruption-Tolerant Networks," in INFOCOM 2006 - The 25th IEEE International Conference on Computer Communications, Barcelona, Catalunya, Spain, April 23-29, 2006, pp. 1-11.

[13] A. Lindgren, A. Doria, E. Davies, and S. Grasic, "Probabilistic Routing Protocol for Intermittently Connected Networks," draft-irtfdtnrg-prophet-02, March 9, 2009, [Online]. Available: http://tools.ietf.org/html/draft-irtf-dtnrg-prophet-02.

[14] A. Keränen, J. Ott, and T. Kärkkäinen, "The ONE Simulator for DTN Protocol Evaluation," in SIMUTools'09: 2nd International Conference on Simulation Tools and Techniques, Rome, March 2-6, 2009. 